\title{
openheart Natural course of tricuspid regurgitation and prognostic implications
}

\author{
Marwin Bannehr (10 , ${ }^{1,2}$ Christoph Roland Edlinger, ${ }^{1,2,3}$ Ulrike Kahn, ${ }^{1}$ \\ Josephin Liebchen, ${ }^{1}$ Maki Okamoto, ${ }^{1}$ Valentin Hähnel, ${ }^{1,2}$ Victoria Dworok, ${ }^{1,2}$ \\ Fabian Schipmann, ${ }^{1,2}$ Tanja Kücken (D) , ${ }^{1,2}$ Karin Bramlage, ${ }^{4}$ Peter Bramlage, ${ }^{4}$ \\ Anja Haase-Fielitz, ${ }^{1,2,5,6}$ Christian Butter ${ }^{1,2,6}$
}

To cite: Bannehr M Edlinger CR, Kahn U, et al. Natural course of tricuspid regurgitation and prognostic implications. Open Heart 2021;8:e001529. doi:10.1136/ openhrt-2020-001529

Received 21 November 2020 Revised 4 January 2021 Accepted 25 January 2021

Check for updates

(c) Author(s) (or their employer(s)) 2021. Re-use permitted under CC BY-NC. No commercial re-use. See rights and permissions. Published by BMJ.

${ }^{1}$ Cardiology, Immanuel Klinikum Bernau Herzzentrum Brandenburg, Bernau, Germany ${ }^{2}$ Brandenburg Medical School (MHB) Theodor Fontane Neuruppin, Germany ${ }^{3}$ Cardiology, Clinic of Internal Medicine II, Paracelsus Medical University of Salzburg, Salzburg, Austria

${ }^{4}$ Institute for Pharmacology and Preventive Medicine, Cloppenburg, Germany ${ }^{5}$ Institute of Social Medicine \& Health Care System Research, Otto-von-Guericke University Magdeburg, Magdeburg, Germany

${ }^{6}$ Faculty of Health Sciences Brandenburg, Potsdam, Germany

Correspondence to Dr Marwin Bannehr; marwin@ bannehr.com

\section{ABSTRACT}

Objective Functional tricuspid regurgitation (TR) is a frequent finding in echocardiography. Literature suggests significant TR is associated with poor prognosis. Still, data remain limited. This study aimed to evaluate long-term prognostic implications in patients with TR.

Methods In this observational cohort study, data from 1650 consecutive patients were analysed. Primary endpoint was all-cause mortality. Mean follow-up time was 1090 days. TR grades at baseline and follow-up were compared. Survival analyses were performed to identify prognostic factors.

Results At baseline, $14.1 \%$ patients showed no, $63.8 \%$ mild, $17.4 \%$ moderate and $4.7 \%$ severe TR. 359 patients (21.8\%) died within the study period. TR at baseline was associated with excess mortality. Moderate and severe TR were of prognostic implication in all subgroups irrespective of systolic pulmonary artery pressure (SPAP) $(</ \geq 40 \mathrm{~mm}$ $\mathrm{Hg}$ ) and left ventricular ejection fraction (LV-EF) $(</ \geq 50 \%)$. Survival was worst in patients with moderate and severe TR and concomitant elevated SPAP or reduced LV-EF at 1 and 3years, respectively $(p<0.001 ; p<0.001)$. In a multivariate model, including cardiac and non-cardiac risk factors, moderate and severe TR, sPAP and impaired right ventricular (RV) function were independent predictors for survival (HR 1.89, Cl 1.07 to 3.36, p=0.029; HR 2.93, Cl 1.57 to $5.49, \mathrm{p}=0.001$; HR 1.44, Cl 1.25 to $1.65, \mathrm{p}<0.001$; HR $1.43, \mathrm{Cl} 1.14$ to $1.79, \mathrm{p}=0.002)$. Overall progression of TR on follow-up was $28.4 \%$. Patients with TR progression showed significantly worse survival ( $\mathrm{HR} 1.44, \mathrm{Cl} 1.11$ to $1.81 ; p=0.006$ )

Conclusion While TR progressed over time, it was associated with impaired long-term survival. TR grade, RV dysfunction, sPAP and TR progression were independent predictors for survival.

\section{INTRODUCTION}

Functional tricuspid regurgitation (TR) is a frequent finding in echocardiography. It is mostly secondary due to left-sided heart disease and occurs predominantly in patients with mitral and aortic valve pathologies. ${ }^{12}$ Besides that, there is evidence that atrial fibrillation (Afib) and right ventricular (RV) pacing may also be of pathophysiological importance..$^{3-5}$ While significant reflux may be well tolerated

\section{Key questions}

What is already known about this subject?

- Literature suggests significant tricuspid regurgitation (TR) is associated with poor prognosis. Still, data remain limited. Particularly quantitative assessment of TR and right ventricular (RV) parameters are lacking in large trials investigating survival.

What does this study add?

- This is the first study to assess TR and its natural course according to quantitative echocardiographic measures, including measurement of TR and the right ventricle in particular. We identified TR grade, RV dysfunction, pulmonary pressure and TR progression as independent predictors for survival besides known risk factors such as left ventricular ejection fraction, NT-proBNP, aortic stenosis, type 2 diabetes and atrial fibrillation. Our findings imply that moderate-to-severe TR robustly associates with mortality and confirm that TR is of prognostic value.

How might this impact on clinical practice?

- As TR is a potentially treatable pathology and progression might be preventable, a more aggressive management may improve patient outcomes, which is of interest since tricuspid valve interventions are on the rise.

- Accurate and standardised echocardiographic assessment of the tricuspid valve and the right ventricle is of prognostic relevance.

for years TR is often neglected. However, literature suggests significant TR is associated with poor prognosis. ${ }^{67}$ Still, data remain limited. Particularly quantitative assessment of TR and RV parameters are lacking in large trials investigating survival.

Quantitative assessment of TR, especially proximal isovelocity surface area (PISA) and effective regurgitant orifice area (EROA) measurement, has recently been shown to be a more powerful and independent predictor for evaluation of TR compared with qualitative assessment by jet area and should be therefore included in the standard assessment. ${ }^{2}$ The prognostic value of PISA in the 
evaluation of clinical outcome in TR patients remains unclear.

Furthermore, it is unknown whether TR itself or rather TR in combination with other comorbidities such as RV dysfunction, pulmonary hypertension (PH), left heart failure or arrhythmias account for an increase in mortality. ${ }^{26}$

Current guidelines for treatment of patients with TR are cautious due to contradictory study results. ${ }^{28}$ At present, only diuretics represent a conservative therapeutic option for volume relief. Surgical treatment has not shown promising results and is primarily recommended in patients undergoing open-heart surgery for mitral and aortic valve disease. ${ }^{9}$ Transcatheter solutions may be a promising approach in the future, still devices are in development and outcome on symptom relief and survival largely unclear ${ }^{10}$

Also, the natural course of TR is not very well known. It has been shown to progress over time. TR development appears to be associated with an increase in pulmonary artery pressures, Afib and coronary artery disease. ${ }^{11}$ Prognostic implications remain largely unclear.

This study aimed to assess TR and its natural course according to quantitative echocardiographic measures in clinical practice and long-term prognostic implications including possible risk factors for adverse outcomes such as RV dysfunction, $\mathrm{PH}$ and left heart failure.

\section{METHODS}

\section{Patient population}

In this retrospective observational cohort study, data from 1650 consecutive patients $(42.8 \%$ female) undergoing echocardiography at two time points at a tertiary care centre in Brandenburg, Germany, from January 2010 to December 2016 were analysed. Follow-up was conducted from June 2018 to June 2019. The patient flow is shown in figure 1.

\section{Data collection and outcome definition}

Demographics and clinical data including relevant comorbidities and laboratory results were collected using patient medical records. Follow-up data for survival rates were collected from the hospital database and primary care physicians. Patients were followed for all-cause mortality.

Echocardiographic examinations were performed with commercially available ultrasound systems (GE-Vingmed, Vivid 7 and E9, Horten Norway) by trained physicians. Complete echocardiograms included 2-dimensional, pulsed-wave, continuous-wave and colour Doppler imaging. The original datasets were prospectively reanalysed. Investigators were blinded for all endpoints to avoid bias. Standard echocardiographic measurements for the evaluation of left atrial size and left ventricular (LV) geometry and function were performed according to the guidelines of the American Society of Echocardiography. ${ }^{12}$ TR determination was conducted in line with current recommendations and guidelines using the following criteria: dilated annulus with

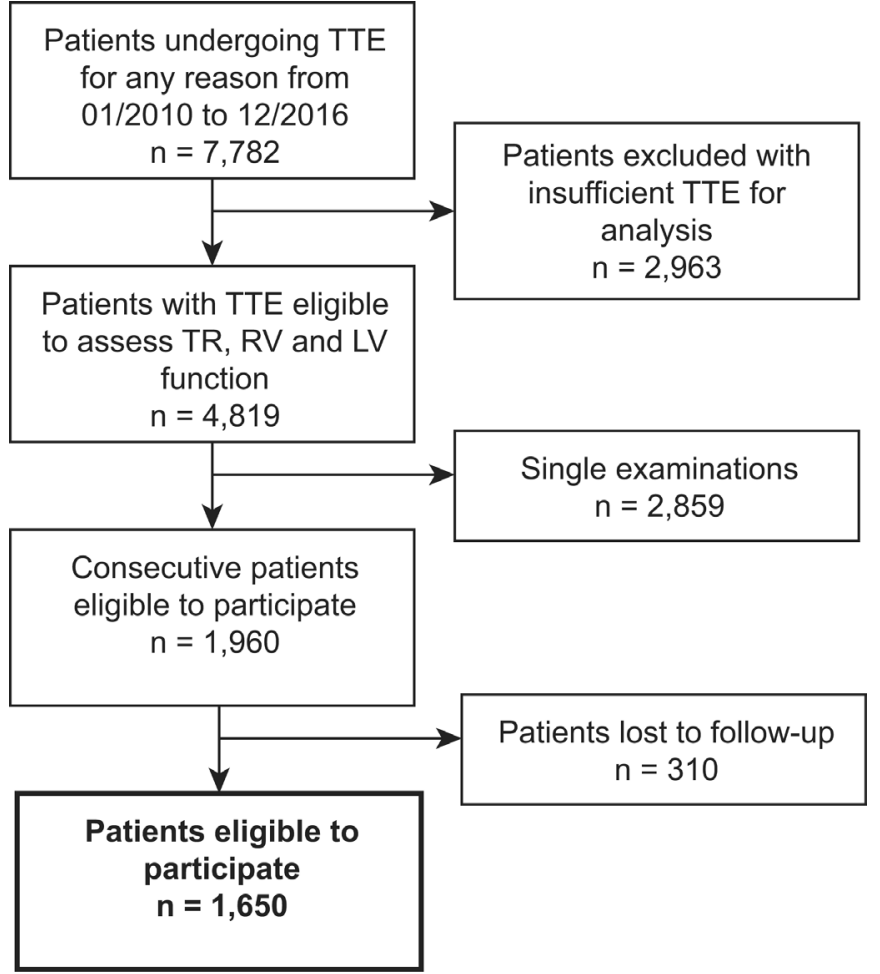

Figure 1 Patient flow through the study. Figure shows patients included and excluded in the study with a number of $n$ individuals at each step. LV, left ventricular; RV, right ventricular; TR, tricuspid regurgitation; TTE, transthoracic echocardiography.

no valve coaptation or flail leaflet, large central jet $>50 \%$ of right atrial, vena contracta $(\mathrm{VC})$ width $>7 \mathrm{~mm}$, PISA radius $>9 \mathrm{~mm}$ at Nyquist $30-40 \mathrm{~cm} / \mathrm{s}$, dense triangular continuous wave jet or sine wave pattern and dilated RV. PISA/EROA measurement was employed including angle correction. PISA and VC were obtained from an apical four chamber zoom view. TR was graded in four stages: none $(0)$, mild (1), moderate, (2) and severe (3). ${ }^{13}$ On a 2D RV focused four chamber view RV size and RV function were analysed. Tricuspid annular plane systolic excursion (TAPSE) was calculated on M-mode recordings. RV function was measured using TAPSE, RV fractional area change (FAC) and tricuspid annular systolic velocity as described previously. ${ }^{15}$ RV dilation was assessed by RV diameters (basal, mid and longitudinal) and RV end-diastolic area in four chamber view. ${ }^{12} 16$

The same views were used for baseline and follow-up examinations. EchoPAC V.202 (GE-Vingmed, Horten, Norway) was used for all echocardiographic analyses.

PH was determined by echocardiographic measurement of TR Vmax and graded into unlikely, possible and likely according to the guidelines of the European Society of Cardiology. ${ }^{17} 18$

Chronic kidney disease (CKD) was defined according to kidney damage with glomerular filtration rate (GFR) $<60 \mathrm{~mL} / \mathrm{min} / 1.73 \mathrm{~m}^{2}$ using CKD-EPI creatinine equation for estimation, irrespective of cause. ${ }^{19} 20$ 
Age-adjusted N-terminal probrain natriuretic peptide (NT-proBNP) cut-offs were determined in accordance with the recommendations of the Heart Failure Association of the European Society of Cardiology. ${ }^{21}$

The primary endpoint was defined as all-cause mortality; the secondary endpoint was progression of TR at follow-up.

The Strengthening the Reporting of Observational Studies in Epidemiology recommendations for reporting observational studies were applied. ${ }^{22}$

The public was involved in the design of this research. Outcome measures and methods of recruitment were discussed in a focus group session.

\section{Statistics}

Data were analysed using descriptive statistics, with categorical variables presented as absolute values and frequencies (\%) and continuous variables presented as means with SD. Comparisons between the four TR grade groups were carried out using an analysis of variance for continuous variables, Fisher's exact or $\chi^{2}$ test for categorical variables.

For the analysis of differences regarding TR grades at baseline and follow-up, TR grade comparisons between these groups were carried out using Wilcoxon test. Survival analysis data are presented as Kaplan-Meier curves. Coxregression analysis with backwards elimination (using a $\mathrm{p}$ value $>0.100$ as removal criterion) for all-cause mortality including cardiac and non-cardiac risk factors was performed. Risk factors included in the analysis were: TR grade, systolic pulmonary artery pressure (sPAP), RV function, TR progression, CKD stage, LV-EF, Afib, age, sex, body mass index (BMI), aortic stenosis, mitral regurgitation, coronary artery disease, hypertension, dyslipidaemia, type 2 diabetes and implantable cardiac device (pacemaker or defibrillator). Results are presented as HRs with CIs.

A p value of $\leq 0.05$ was considered statistically significant.

All statistical tests were performed using IBM SPSS Statistics software V.24.0 or V.26.0 (IBM Corporation).

\section{RESULTS}

\section{Baseline characteristics}

Of all 1650 consecutive patients (42.8\% male), 232 (14.1 $\%)$ patients showed no, $1053(63.8 \%)$ mild, $287(17.4 \%)$ moderate and $78(4.7 \%)$ severe functional TR at baseline. On average, patients were obese (BMI $27.9 \pm 5.1 \mathrm{~kg} /$ $\mathrm{m}^{2}$ ) and showed a relevant number of cardiovascular risk factors, cardiac and general comorbidities (table 1).

Higher TR grades were found when patients were at an advanced age (TR $070.6 \pm 12.8$ years vs TR $173.0 \pm 10.8$ years, TR $275.7 \pm 9.3$ years and TR $374.5 \pm 9.9$ years; $\mathrm{p}<0.001$ ) or of female sex (TR $037.5 \%$ vs TR $140.9 \%$, TR 2 50.7\% and TR $355.1 \%$; p=0.001). Patients with moderate and severe TR showed a trend towards a lower BMI (TR $029.8 \pm 5.5 \mathrm{~kg} / \mathrm{m}^{2}$ vs TR $127.9 \pm 4.9 \mathrm{~kg} / \mathrm{m}^{2}$, TR 2 $26.7 \pm 5.1 \mathrm{~kg} / \mathrm{m}^{2}$ and TR $\left.326.9 \pm 5.0 ; \mathrm{p}<0.001\right)$.
$\mathrm{PH}$ according to echocardiographic criteria was more likely in patients with TR compared with those with none (TR $04.7 \%$ vs TR $118.8 \%$, TR 248.4 and TR 3 29.5\%; $\mathrm{p}<0.001)$. With increasing TR severity, there was an incline in the incidence of Afib (TR $025.9 \%$ vs TR $144.7 \%$, TR $270.3 \%$ and TR 3 80.5\%; $\mathrm{p}<0.001)$. Aortic stenosis, mitral regurgitation and dyslipidaemia were less often detected in patients with TR, while prior implantation of a cardiac device (pacemaker or defibrillator), prior mitral valve surgery and CKD were more likely (table 1).

Patients with TR showed significantly higher NT-proBNP levels (no TR $1264 \pm 1783 \mathrm{pg} / \mathrm{mL}$ vs mild TR $3461 \pm 4695 \mathrm{pg} / \mathrm{mL}$, moderate TR $5264 \pm 5025 \mathrm{pg} / \mathrm{mL}$ and severe TR $6029 \pm 6689 \mathrm{pg} / \mathrm{mL} ; \mathrm{p}<0.001)$ and worse CKD-EPI GFR (no TR $71.0 \pm 29.3 \mathrm{~mL} / \mathrm{min}$ vs mild TR $65.6 \pm 26.4 \mathrm{~mL} / \mathrm{min}$, moderate TR $56.4 \pm 27.0 \mathrm{~mL} / \mathrm{min}$ and severe TR $55.5 \pm 21.3 \mathrm{~mL} / \mathrm{min} ; \mathrm{p}<0.001)$ compared with those with no TR (table 1).

TR PISA, EROA and VC were significantly higher in patients with TR compared with no TR as baseline (all $\mathrm{p}<0.001)$. Tricuspid valve (TV) annular diameters $(\mathrm{p}<0.001)$ and RV size increased with TR grade (RV basal $\mathrm{p}<0.001, \mathrm{RV}$ mid $\mathrm{p}<0.001$, RV longitudinal $\mathrm{p}=0.009$, RV end-diastolic area $\mathrm{p}<0.001)$. Patients with moderate or severe TR showed significantly reduced RV function compared no TR (TAPSE $\mathrm{p}<0.001$, RV FAC $\mathrm{p}<0.001$, TASV $p<0.001)$. TR was also associated with impaired LV function $(\mathrm{p}<0.001)$.

Echocardiographic characteristics overall and divided by TR grade are shown in table 2 .

\section{Long-term survival}

A total of 359 patients $(21.8 \%)$ died within the study period. Mean follow-up time was 1090 days. TR on baseline echocardiography was associated with excess mortality in Kaplan-Meier analysis (log rank, $\mathrm{p}<0.001$ ) (figure 2A). Moderate and severe TR were of prognostic implication in all subgroups irrespective of $\mathrm{sPAP}(</ \geq 40 \mathrm{~mm} \mathrm{Hg})$ and LV-EF $(</ \geq 50 \%)$ (figure 2B-E). Survival was worst in patients with moderate and severe TR and concomitant elevated sPAP or reduced LV-EF $(\mathrm{p}<0.001 ; \mathrm{p}<0.001)$ (figure 2B-E).

In a multivariate model, including cardiac and noncardiac risk factors, moderate and severe TR, sPAP $(\geq 40 \mathrm{~mm} \mathrm{Hg}$ ) and impaired $\mathrm{RV}$ function (TAPSE $\geq 18.5 \mathrm{~mm}$ ) were independent risk factors for survival (HR 1.89, CI 1.07 to 3.36, $\mathrm{p}=0.029$; HR 2.93, CI 1.57 to 5.49, $\mathrm{p}=0.001$; HR 1.44, CI 1.25 to 1.65, $\mathrm{p}<0.001$; HR 1.43, CI 1.14 to $1.79, \mathrm{p}=0.002$ ) besides CKD, impaired LV-EF, NT-proBNP, aortic stenosis, type 2 diabetes and Afib (table 3).

\section{Progression of TR}

Compared with baseline echocardiographic assessment, there was significant overall progression of TR on follow-up in $28.4 \%$ of patients within the study period (at least one grade on echocardiography). TR progressed stronger over time but was only detectable after at least 


\begin{tabular}{|c|c|c|c|c|c|c|}
\hline & $\begin{array}{l}\text { Total } \\
\mathrm{N}=1650\end{array}$ & $\begin{array}{l}\text { TR } 0 \\
\mathrm{~N}=232\end{array}$ & $\begin{array}{l}\text { TR 1 } \\
\mathrm{N}=1053\end{array}$ & $\begin{array}{l}\text { TR 2 } \\
\mathrm{N}=287\end{array}$ & $\begin{array}{l}\text { TR } 3 \\
\mathrm{~N}=78\end{array}$ & \\
\hline & Mean $\pm S D$ or $\%$ & Mean \pm SD or $\%$ & Mean \pm SD or $\%$ & Mean \pm SD or $\%$ & Mean \pm SD or $\%$ & $P$ value \\
\hline Age in years & $73.2 \pm 10.9$ & $70.6 \pm 12.8$ & $73.0 \pm 10.8$ & $75.7 \pm 9.3$ & $74.5 \pm 9.9$ & $<0.001$ \\
\hline Female gender & 42.8 & 37.5 & 40.9 & 50.7 & 55.1 & 0.001 \\
\hline BMI $\left(\mathrm{kg} / \mathrm{m}^{2}\right)$ & $27.9 \pm 5.1$ & $29.8 \pm 5.5$ & $27.9 \pm 4.9$ & $26.7 \pm 5.1$ & $26.9 \pm 5.0$ & $<0.001$ \\
\hline \multicolumn{7}{|l|}{ CV risk factors } \\
\hline Hypertension & 92.7 & 91.7 & 93.0 & 92.3 & 93.5 & 0.892 \\
\hline Dyslipidaemia & 69.8 & 73.2 & 71.3 & 64.7 & 58.4 & 0.013 \\
\hline \multicolumn{7}{|l|}{ Comorbidities general } \\
\hline Diabetes mellitus & 33.5 & 38.3 & 31.4 & 35.3 & 39.0 & 0.107 \\
\hline CKD (eGFR <60 mL/min) & 45.6 & 32.6 & 44.2 & 56.8 & 61.5 & $<0.001$ \\
\hline COPD & 15.5 & 12.3 & 14.8 & 18.5 & 23.4 & 0.051 \\
\hline \multicolumn{7}{|l|}{ Comorbidities cardiac } \\
\hline \multicolumn{7}{|l|}{ Pulmonary hypertension } \\
\hline Unlikely* & 51.5 & 88.8 & 53.0 & 18.1 & 42.3 & $<0.001$ \\
\hline Possible $^{\star}$ & 26.1 & 6.5 & 28.2 & 33.4 & 28.2 & \\
\hline Likely* & 22.5 & 4.7 & 18.8 & 48.4 & 29.5 & \\
\hline Atrial fibrillation & 48.3 & 25.9 & 44.7 & 70.3 & 80.5 & $<0.001$ \\
\hline DCM & 10.7 & 6.6 & 10.8 & 13.0 & 13.0 & 0.109 \\
\hline Coronary artery disease & 57.9 & 57.8 & 59.8 & 53.8 & 48.1 & 0.089 \\
\hline Aortic stenosis (at least moderate) & 19.5 & 20.3 & 21.5 & 13.9 & 11.5 & $<0.001$ \\
\hline Prior aortic valve replacement & 9.1 & 10.3 & 8.2 & 11.8 & 7.7 & 0.226 \\
\hline Mitral regurgitation (at least moderate) & 18.8 & 3.0 & 13.8 & 53.2 & 54.9 & $<0.001$ \\
\hline Prior mitral valve replacement/repair & 4.6 & 2.6 & 3.8 & 8.0 & 9.0 & 0.003 \\
\hline Prior cardiac device & 22.6 & 10.5 & 21.5 & 32.9 & 35.1 & $<0.001$ \\
\hline \multicolumn{7}{|l|}{ Laboratory results } \\
\hline NT-proBNP $(n=995)$ & $3610 \pm 4766$ & $1264 \pm 1783$ & $3461 \pm 4695$ & $5264 \pm 5025$ & $6209 \pm 6689$ & $<0.001$ \\
\hline CKD-EPI GFR (ml/min) $(\mathrm{n}=1244)$ & $64.4 \pm 27.1$ & $71.0 \pm 29.3$ & $65.6 \pm 26.4$ & $56.4 \pm 27.0$ & $55.5 \pm 21.3$ & $<0.001$ \\
\hline
\end{tabular}

Bold values denote statistical significance at the $p \leq 0.05$ level.

*The probability of pulmonary hypertension was estimated by the peak tricuspid regurgitation velocity and graded according to European Society of Cardiology guidelines. ${ }^{17} 18$

$\mathrm{BMI}$, body mass index; CV, cardiovascular; CKD, chronic kidney disease; eGFR, estimated glomerular filtration rate; COPD, chronic obstructive pulmonary disease; DCM, dilative cardiomyopathy; NT-proBNP, N-terminal pro brain natriuretic peptide.;

1 year of follow-up $(\leq 1$ year $\mathrm{p}=0.310 ;>1$ year -3 years $\mathrm{p}=0.033$, >3-6years $\mathrm{p}<0.001$ and $>6-9$ years $\mathrm{p}<0.001$; figure 3).

Patients with TR progression showed significantly worse survival (HR 1.44, CI 1.11 to $1.81 ; \mathrm{p}=0.006$; table 3 and figure 4$)$.

\section{DISCUSSION}

In this study, we assessed TR and its natural course according to quantitative echocardiographic measures in clinical practice and evaluated the long-term prognostic implications including possible risk factors.

Within our patient population, almost $25 \%$ of patients exhibited moderate or severe TR. Moderate and severe TR had prognostic value irrespective of sPAP and LV-EF. However, survival was lowest in patients with concomitant elevated sPAP and reduced LV-EF. On follow-up, there was significant progression of TR in approximately $30 \%$ of patients.

Besides TR grade, RV dysfunction, sPAP, TR progression, LV-EF, NT-proBNP, CKD, aortic stenosis, type 2 diabetes and Afib were independent predictors for survival.

Patients with significant TR were more likely at an advanced age and female. They exhibited Afib, PH and CKD more often and were more prone to have a history of prior mitral valve surgery or implantation of a cardiac device.

\section{Clinical implications}

TR is a common finding in echocardiography and considered benign at first. ${ }^{12}$ Initially, symptoms are nonspecific 
Table 2 Echocardiographic parameters at baseline echocardiography

\begin{tabular}{|c|c|c|c|c|c|c|}
\hline & $\begin{array}{l}\text { Total } \\
\mathrm{N}=1650\end{array}$ & $\begin{array}{l}\text { TRO } \\
\mathrm{N}=232 \\
\end{array}$ & $\begin{array}{l}\text { TR1 } \\
\mathrm{N}=1053\end{array}$ & $\begin{array}{l}\text { TR2 } \\
\mathrm{N}=287 \\
\end{array}$ & $\begin{array}{l}\text { TR3 } \\
\mathrm{N}=78 \\
\end{array}$ & \\
\hline & Mean \pm SD & Mean \pm SD & Mean \pm SD & Mean \pm SD & Mean \pm SD & $P$ value \\
\hline TR PISA (cm) & $0.5 \pm 0.2$ & $0.0 \pm 0.0$ & $0.4 \pm 0.2$ & $0.7 \pm 0.2$ & $0.9 \pm 0.3$ & $<0.001$ \\
\hline TR EROA $\left(\mathrm{mm}^{2}\right)$ & $0.31 \pm 0.32$ & $0.0 \pm 0.0$ & $0.18 \pm 0.13$ & $0.38 \pm 0.22$ & $0.82 \pm 0.68$ & $<0.001$ \\
\hline TR vena contracta $(\mathrm{mm})$ & $4.7 \pm 2.4$ & $0.0 \pm 0.0$ & $3.8 \pm 1.7$ & $5.5 \pm 1.9$ & $8.0 \pm 2.9$ & $<0.001$ \\
\hline TV annulus (mm) & $30.9 \pm 6.5$ & $28.6 \pm 5.3$ & $30.0 \pm 6.1$ & $33.8 \pm 6.4$ & $37.3 \pm 7.2$ & $<0.001$ \\
\hline sPAP $(\mathrm{mm} \mathrm{Hg})$ & $36.0 \pm 13.9$ & $26.6 \pm 12.0$ & $34.3 \pm 12.4$ & $43.7 \pm 14.0$ & $36.8 \pm 18.2$ & $<0.001$ \\
\hline $\mathrm{TR} \operatorname{Vmax}(\mathrm{m} / \mathrm{s})$ & $2.9 \pm 1.6$ & $2.5 \pm 0.6$ & $2.9 \pm 0.5$ & $3.3 \pm 0.5$ & $2.9 \pm 0.7$ & 0.051 \\
\hline TAPSE $(\mathrm{mm})$ & $20.0 \pm 5.2$ & $22.5 \pm 5.2$ & $20.4 \pm 5.1$ & $17.8 \pm 4.5$ & $17.2 \pm 5.0$ & $<0.001$ \\
\hline TASV (mm) & $11.3 \pm 3.2$ & $13.2 \pm 3.1$ & $11.4 \pm 3.2$ & $10.2 \pm 2.8$ & $10.3 \pm 2.6$ & $<0.001$ \\
\hline LV-EF (\%) & $46.8 \pm 14.3$ & $52.5 \pm 11.0$ & $47.1 \pm 14.2$ & $43.0 \pm 15.4$ & $42.6 \pm 14.8$ & $<0.001$ \\
\hline LVEDD (mm) & $51.2 \pm 9.1$ & $49.2 \pm 8.2$ & $51.1 \pm 9.0$ & $53.1 \pm 9.5$ & $51.8 \pm 9.7$ & $<0.001$ \\
\hline LVESD (mm) & $40.0 \pm 10.6$ & $38.1 \pm 9.5$ & $39.9 \pm 10.5$ & $41.8 \pm 11.1$ & $40.1 \pm 11.5$ & 0.002 \\
\hline $\mathrm{RV} 1(\mathrm{~mm})$ & $37.1 \pm 7.5$ & $33.1 \pm 6.0$ & $36.3 \pm 6.9$ & $41.0 \pm 7.1$ & $44.5 \pm 8.1$ & $<0.001$ \\
\hline $\mathrm{RV} 2(\mathrm{~mm})$ & $26.6 \pm 7.0$ & $24.8 \pm 6.5$ & $25.9 \pm 6.7$ & $28.7 \pm 7.1$ & $32.4 \pm 7.6$ & $<0.001$ \\
\hline RV3 (mm) & $66.0 \pm 10.2$ & $67.3 \pm 10.4$ & $65.4 \pm 10.0$ & $66.5 \pm 10.5$ & $68.8 \pm 10.4$ & 0.009 \\
\hline RV FAC (\%) & $37.4 \pm 18.8$ & $39.9 \pm 17.4$ & $38.3 \pm 20.4$ & $33.1 \pm 13.3$ & $34.5 \pm 12.0$ & $<0.001$ \\
\hline RV area end diastolic $\left(\mathrm{cm}^{2}\right)$ & $22.3 \pm 10.4$ & $22.1 \pm 10.9$ & $21.5 \pm 10.2$ & $24.0 \pm 10.0$ & $28.8 \pm 10.6$ & $<0.001$ \\
\hline MPI Index (\%) & $0.5 \pm 0.2$ & $0.5 \pm 0.2$ & $0.5 \pm 0.2$ & $0.5 \pm 0.2$ & $0.6 \pm 0.3$ & 0.204 \\
\hline
\end{tabular}

Bold values denote statistical significance at the $p \leq 0.05$ level

EROA, effective regurgitant orifice area; FAC, fractional area change; LVEDD, left ventricular end-diastolic diameter; LV-EF, left ventricular ejection fraction; LVESD, left ventricular end-systolic diameter; MPI, myocardial performance index; ;PISA, proximal isovelocity surface area; RV, right ventricular, 1 basal, 2 mid, 3 longitudinal; sPAP, systolic pulmonary artery pressure; TAPSE, tricuspid annular plane systolic excursion; TASV, tricuspid annular systolic velocity; TR, tricuspid regurgitation; TV, tricuspid valve.

and progress slowly. Patients at later stages, who present with relevant TR and heart failure, often already show signs of irreversible RV dysfunction.

In our study, moderate-to-severe TR was associated with increased mortality throughout all patient subgroups, including normal and impaired LV-EF as well as normal and increased pulmonary pressure. However, survival was worst in those patients with reduced LV-EF and elevated pulmonary pressure.

This is the first study to assess TR and its natural course according to quantitative measurement of $\mathrm{TR}$ and $\mathrm{RV}$ in particular, showing that TR is of prognostic value irrespective of sPAP and LV-EF in a large patient cohort. It confirms the results of Nath et al and Benfari et al, that moderate and severe TR are associated with a poor prognosis. ${ }^{67}$ However, Nath et al only qualitatively graded TR by jet area, which is less robust. Quantitative assessment may have been helpful, as the authors state. Also, RV enlargement was considered primarily in comparison to LV size without definite measurements. RV function was mainly evaluated by TAPSE only. Most of patients were men and clinical characteristics were unknown. ${ }^{7}$ The other study by Benfari et al only addressed patients with heart failure and impaired LV function, that is, mean LV-EF $36 \%$, which only represents a limited subgroup of patients at risk. ${ }^{6}$
Moderate-to-severe TR is associated with annular dilation. Pathophysiologically, functional TR cannot be considered a mere valvular disease. It should rather be regarded in the context of RV dysfunction. Increased RV afterload, that is, elevated pulmonary pressure by either LV dysfunction or left-sided valvular disease, cause RV and tricuspid annular dilation and thus TR. ${ }^{23}$ Patients with relevant TR showed significantly dilated RV and impaired RV function in this cohort. Hemodynamically, a 'vicious circle' of RV dilatation, RV dysfunction and TR evolves over the course of time. Previous studies have shown an association between RV dilation and increased mortality. ${ }^{24}$ At the same time, TR is commonly associated with RV enlargement. However, the reason for excess mortality in patients with TR remains to be determined.

Interestingly, almost half of patients with severe $\mathrm{PH}$ showed only mild TR. ${ }^{25}$ These findings underline difficulties in echocardiographic assessment of right heart function including TR and PH. In this context, new imaging techniques represent a spectrum of high interest. In the future, the use of new echocardiographic methods such as RV strain using speckle tracking may allow a much more precise assessment of the RV.

Mild TR in structurally heart-healthy individuals is considered a physiological and not a pathological finding. In this study, we were able to identify a significant overall 

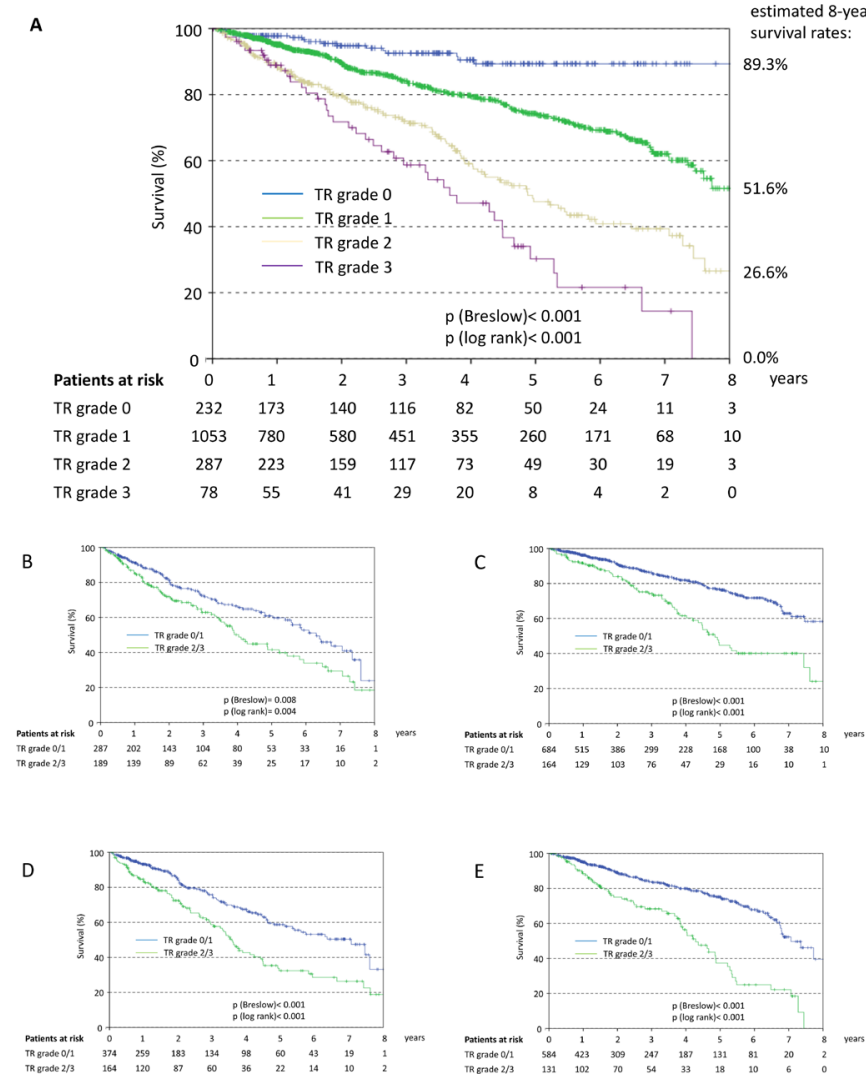

Figure 2 Kaplan-Meier survival curve for (A) all patients divided by TR grade and patients separated by TR grade 0/1 versus $2 / 3$ and (B) sPAP $\geq 40 \mathrm{mmHg}$, (C) sPAP $<40 \mathrm{~mm} \mathrm{Hg}$, (D) LV-EF $<50 \%$, and (E) LV-EF $\geq 50 \%$. Patients with TR show significantly worse survival. Moderate and severe TR had prognostic value irrespective of sPAP $(</ \geq 40 \mathrm{~mm} \mathrm{Hg})$ and LVEF $(</ \geq 50 \%)$. However, survival was lowest in patients with concomitant elevated SPAP and reduced LV-EF.

progression of TR on follow-up. Based on our results, a time interval of at least 1 year is necessary to detect a significant change on echocardiography. This seems to be important for planning routine follow-ups, as relevant progression is likely over time. Furthermore, patients, who suffer from fast development of TR, have been shown to have worse survival than those with slower development. ${ }^{11}$ In this context, large prospective studies, ideally with knowledge of the invasive pressure gradients, would be necessary to find a sustainable therapeutic approach to break the cycle of RV dysfunction and TR.

Increased NT-proBNP were associated with excess mortality in this study. However, unspecific regarding the cause of heart failure, the marker may add additional information with prognostic implications in patients with TR.

Interestingly, there were several significant differences between patient groups with regard to TR grade, that is, gender, BMI and prior implantation of cardiac devices, which did not appear to be independent risk factors for mortality.

Women showed a trend towards higher TR grades in this cohort with regard to baseline characteristics, even though gender was insignificant in the multivariate survival model. According to current literature, the incidence of heart failure is generally lower in women than in men at all ages. Due to an increase in incidence of heart failure with age and a proportionally larger number of elderly women, the total number of men and women living with heart failure has been overall reported as similar. $^{26}$

Patients with RV dysfunction may furthermore show signs of cardiac cachexia. This has been associated with adverse effects in the past and reported as a relevant risk factor in patients undergoing valvular surgery indicating end-staged disease. ${ }^{2728} \mathrm{~A}$ decreasing BMI with TR progression in our study population might indicate a pathological shift of metabolism towards a rather catabolic state. Despite this negative trend, it is noteworthy that even in patients with severe TR mean BMI was $>25 \mathrm{~kg} / \mathrm{m}^{2}$.

There has been growing evidence of interplay regarding Afib, TV annular and right atrial dilation and remodelling with consequent functional TR. ${ }^{4} \mathrm{On}$ the one hand, restoration of sinus rhythm has been associated with reverse remodelling of the right atrium and improvement of TR severity. ${ }^{3}$ Still, data are limited. On the other hand, atrial enlargement caused by atrioventricular valve reflux is a well-known risk factor for Afib itself. ${ }^{29}$

Chronic RV pacing may further deteriorate RV (and LV) function causing pacing-induced cardiomyopathy and therefore worsen TR. ${ }^{30} \mathrm{RV}$ lead interference can mechanically cause TR while there is also evidence for increase in TR grade by RV pacing, irrespective of altered RV function and direct mechanical disruption. ${ }^{5}$ Patients with advanced TR had up to three times more often a history of prior pacemaker or defibrillator implantation, which appears logical because they suffer from progressive heart failure.

Our findings imply that moderate-to-severe TR robustly associates with mortality. We believe that in the future accurate and standardised assessment of the TV and RV is of importance to identify patients at risk. Still further prospective data including possible interventions of the TV are needed in order to establish an ideal treatment of patients with TR. Since TR is a potentially treatable pathology and progression may be preventable, a more aggressive management might improve patient outcomes in the future. At the moment, TR is mainly managed medically by administering diuretics for symptomatic relief. ${ }^{31}$ Surgical isolated TV repair has not been shown to be promising. Indication for TV surgery is limited mainly to patients who simultaneously undergo mitral or aortic valve repair. Referral for surgery in patients with isolated TR may be beneficial at an early stage ${ }^{32}$ Data imply the importance to intervene while the right ventricle has not been irreversibly damaged. ${ }^{33}$ Transcatheter solutions may be a promising approach in the future since they are less invasive. However, all interventional devices are still in development. ${ }^{10}$

\section{Study limitations}

The study was retrospectively performed at a single centre. Lead time bias may be a confounder. Patients underwent echocardiography for a variety of indications. 


\begin{tabular}{|c|c|c|c|}
\hline Variable & HR & $\mathrm{Cl}$ & P value \\
\hline TR grade & & & $<0.001$ \\
\hline 1 & 1.573 & 0.930 to 2.661 & 0.091 \\
\hline 2 & 1.892 & 1.066 to 3.359 & 0.029 \\
\hline 3 & 2.934 & 1.569 to 5.488 & 0.001 \\
\hline CKD (CKD-EPI GFR $<60 \mathrm{~mL} / \mathrm{min}$ ) & 1.602 & 1.283 to 2.001 & $<0.001$ \\
\hline LV systolic function & & & 0.014 \\
\hline Mildly impaired (EF $45-<55 \%$ ) & 1.076 & 0.811 to 1.426 & 0.613 \\
\hline Moderately impaired (EF $35-<45 \%$ ) & 1.421 & 1.040 to 1.942 & 0.027 \\
\hline Severely impaired (EF <35 \%) & 1.563 & 1.128 to 2.166 & 0.007 \\
\hline NT-proBNP (age adjusted cut-offs according to $\mathrm{ESC}^{21}$ ) & 1.479 & 1.151 to 1.900 & 0.002 \\
\hline TR progression ( $\geq 1$ grade) & 1.442 & 1.112 to 1.814 & 0.006 \\
\hline $\operatorname{sPAP}(\geq 40 \mathrm{~mm} \mathrm{Hg})$ & 1.435 & 1.249 to 1.649 & $<0.001$ \\
\hline RV function (TAPSE $<18.5 \mathrm{~mm}$ ) & 1.431 & 1.143 to 1.792 & 0.002 \\
\hline Aortic stenosis (at least moderate) & 1.391 & 1.092 to 1.773 & 0.008 \\
\hline Mitral regurgitation (at least moderate) & 1.283 & 0.981 to 1.678 & 0.068 \\
\hline Type 2 diabetes & 1.258 & 1.015 to 1.558 & 0.036 \\
\hline Afib & 1.262 & 1.003 to 1.588 & 0.047 \\
\hline
\end{tabular}

Cox-regression analysis with backward elimination (using a $p$ value $>0.100$ as removal criterion) for all-cause mortality, including cardiac and non-cardiac risk factors. Risk factors included were: TR grade, CKD, LV systolic function, NT-proBNP, sPAP, RV function, aortic stenosis, mitral regurgitation, type 2 diabetes, Afib, coronary artery disease, hypertension, implantable cardiac device (pacemaker or defibrillator) and sex.

Bold values denote statistical significance at the $p \leq 0.05$ level.

Afib, atrial fibrillation; CKD, chronic kidney disease; ESC, European Society of Cardiology; GFR, glomerular filtration rate; LV, left ventricular; NT-proBNP, N-terminal pro brain natriuretic peptide; RV, right ventricular; sPAP, systolic pulmonary artery pressure; TAPSE, tricuspid annular plane systolic excursion; TR, tricuspid regurgitation.

Early detection of TR in the disease course may artificially inflate perceived survival duration.

While tricuspid, aortic and mitral valve pathologies, $\mathrm{RV}$ and LV function were analysed in all patients at baseline and follow-up, some parameters, such as PH or GFR, could not be reanalysed in all patients but still the vast majority. Only patients with echocardiographic examinations at baseline and follow-up were included.

TR is known to fluctuate and to be load dependent. Standards for evaluation of TR are less robust than for mitral or aortic valve disease. The jet area can have an

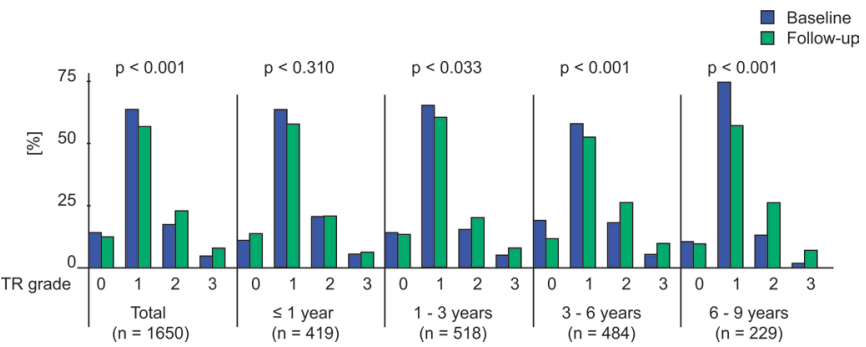

Figure 3 TR progression over time divided by followup (FU) time intervals: Total, $\leq 1$ year, $>1$ to $\leq 3$ years, $>3$ to $\leq 6$ years, and $>6$ to $<9$ years. Compared with baseline echocardiographic assessment, there was significant overall progression of TR on follow-up. TR progressed stronger over time but was only detectable after more than 1 year of FU. overlap in patients with mild versus moderate TR. It can be considered semiquantitative, as the flow in wideopen TR may be so low that aliasing does not occur as a distinct jet. Since imaging of the inferior vena cava and hepatic vein flow were not part of the standard echocardiography protocol, they could not be assessed in this cohort.

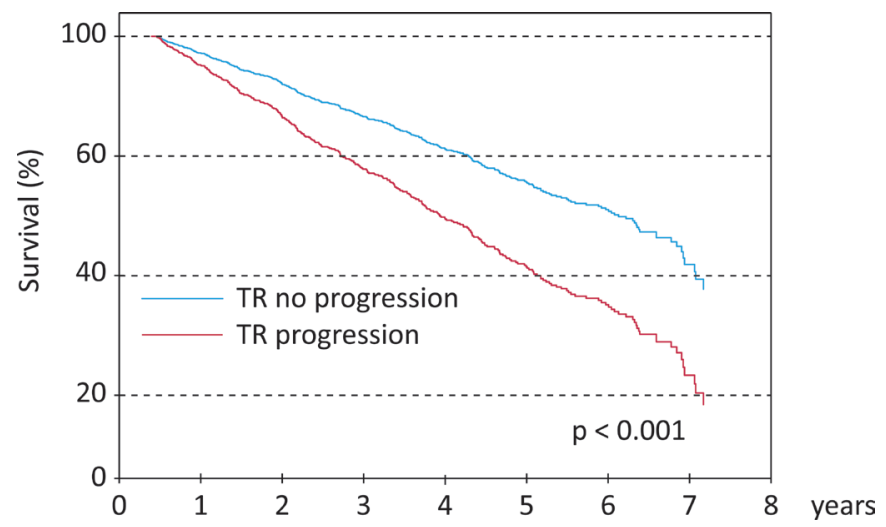

Figure 4 Kaplan-Meier survival curve for patients divided by TR progression (at least one grade) on follow-up echocardiography. Patients with TR progression showed significantly worse survival (HR 1.44, Cl 1.11 to 1.81 ; $p=0.006)$. 
There were no data available for administered medications, especially diuretics.

\section{CONCLUSION}

While TR progressed over time, it was associated with impaired long-term survival in all subgroups. TR grade, RV dysfunction, sPAP and TR progression were independent predictors for survival besides LV-EF, NT-proBNP, aortic stenosis, type II diabetes and Afib.

Contributors MB coordinated the study, acquisited and analysed data, prepared figures and wrote the manuscript. CRE interpreted data, contributed to manuscript preparation and final submission. $\mathrm{UK}, \mathrm{JL}$ and $\mathrm{MO}$ acquisited and analysed data. VH, VD, FS and TK interpreted data and contributed to manuscript preparation. KB and $\mathrm{PB}$ analysed data and revised the article critically for the content. $\mathrm{AH}-\mathrm{F}$ and $\mathrm{CB}$ planned the study. All authors provided final approval of the article.

Funding This research received funding from Deutsche Stiftung für Herzforschung, Bockenheimer Landstr. 94-96, 60323 Frankfurt am Main.

\section{Competing interests None declared.}

Patient consent for publication Not required.

Ethics approval The study was approved by the local Ethics Committee of the State Brandenburg (AS 155(bB)/2017) and was conducted in accordance with the Declaration of Helsinki.

Provenance and peer review Not commissioned; externally peer reviewed.

Data availability statement Original data are available upon reasonable request.

Open access This is an open access article distributed in accordance with the Creative Commons Attribution Non Commercial (CC BY-NC 4.0) license, which permits others to distribute, remix, adapt, build upon this work non-commercially, and license their derivative works on different terms, provided the original work is properly cited, appropriate credit is given, any changes made indicated, and the use is non-commercial. See: http://creativecommons.org/licenses/by-nc/4.0/.

\section{ORCID iDs}

Marwin Bannehr http://orcid.org/0000-0001-9762-4167

Tanja Kücken http://orcid.org/0000-0002-1482-951X

\section{REFERENCES}

1 Dreyfus GD, Martin RP, Chan KMJ, et al. Functional tricuspid regurgitation: a need to revise our understanding. J Am Coll Cardiol 2015;65:2331-6.

2 Topilsky Y, Nkomo VT, Vatury O, et al. Clinical outcome of isolated tricuspid regurgitation. JACC Cardiovasc Imaging 2014;7:1185-94.

3 Muraru D, Caravita S, Guta AC, et al. Functional tricuspid regurgitation and atrial fibrillation: which comes first, the chicken or the egg? CASE 2020;4:458-63.

4 Utsunomiya $\mathrm{H}$, Itabashi $\mathrm{Y}$, Mihara $\mathrm{H}$, et al. Functional tricuspid regurgitation caused by chronic atrial fibrillation: a real-time 3-dimensional transesophageal echocardiography study. Circ Cardiovasc Imaging 2017;10.

5 Vaturi M, Kusniec J, Shapira Y, et al. Right ventricular pacing increases tricuspid regurgitation grade regardless of the mechanical interference to the valve by the electrode. Eur $J$ Echocardiogr 2010;11:550-3.

6 Benfari G, Antoine C, Miller WL, et al. Excess mortality associated with functional tricuspid regurgitation complicating heart failure with reduced ejection fraction. Circulation 2019;140:196-206.

7 Nath J, Foster E, Heidenreich PA. Impact of tricuspid regurgitation on long-term survival. J Am Coll Cardiol 2004;43:405-9.

8 Sonne C. Diagnostik und konservative therapie der Trikuspidalklappeninsuffizienz. CardioVasc 2016;16:45-51.

9 Baumgartner H, Falk V, Bax JJ, et al. 2017 ESC/EACTS guidelines for the management of valvular heart disease. Eur Heart $J$ 2017;38:2739-91.

10 Hahn RT, Zamorano JL. The need for a new tricuspid regurgitation grading scheme. Eur Heart J Cardiovasc Imaging 2017;18:1342-3.

11 Prihadi EA, van der Bijl P, Gursoy E, et al. Development of significant tricuspid regurgitation over time and prognostic implications: new insights into natural history. Eur Heart J 2018;39:3574-81.
12 Lang RM, Badano LP, Mor-Avi V, et al. Recommendations for cardiac chamber quantification by echocardiography in adults: an update from the American Society of echocardiography and the European association of cardiovascular imaging. J Am Soc Echocardiogr 2015;28:1-39. e14.

13 Nishimura RA, Otto CM, Bonow RO, et al. 2014 AHA/ACC guideline for the management of patients with valvular heart disease: Executive summary: a report of the American College of Cardiology/ American heart association Task force on practice guidelines. Circulation 2014;129:2440-92.

14 Joint Task Force on the Management of Valvular Heart Disease of the European Society of Cardiology (ESC), European Association for Cardio-Thoracic Surgery (EACTS), Vahanian A, et al. Guidelines on the management of valvular heart disease (version 2012). Eur Heart $J$ 2012;33:2451-96.

15 Rudski LG, Lai WW, Afilalo J, et al. Guidelines for the echocardiographic assessment of the right heart in adults: a report from the American Society of echocardiography endorsed by the European association of echocardiography, a registered branch of the European Society of cardiology, and the Canadian Society of echocardiography. J Am Soc Echocardiogr 2010;23:685-713. quiz 86-8.

16 Muraru D, Onciul S, Peluso D, et al. Sex- and Method-Specific reference values for right ventricular strain by 2 -dimensional Speckle-Tracking echocardiography. Circ Cardiovasc Imaging 2016;9:e003866-e.

17 Galiè N, Hoeper MM, Humbert M, et al. Guidelines for the diagnosis and treatment of pulmonary hypertension: the task force for the diagnosis and treatment of pulmonary hypertension of the European Society of cardiology (ESC) and the European respiratory Society (ERS), endorsed by the International Society of heart and lung transplantation (ISHLT). Eur Heart $J$ 2009;30:2493-537.

18 Galiè N, Humbert M, Vachiery J-L, et al. 2015 ESC/ERS guidelines for the diagnosis and treatment of pulmonary hypertension: the joint Task force for the diagnosis and treatment of pulmonary hypertension of the European Society of cardiology (ESC) and the European respiratory Society (ERS): endorsed by: association for European paediatric and congenital cardiology (AEPC), International Society for heart and lung transplantation (ISHLT). Eur Heart $J$ 2016;37:67-119.

19 Levey AS, Eckardt K-U, Tsukamoto Y, et al. Definition and classification of chronic kidney disease: a position statement from kidney disease: improving global outcomes (KDIGO). Kidney Int 2005;67:2089-100.

20 Levey AS, Stevens LA. Estimating GFR using the CKD epidemiology collaboration (CKD-EPI) creatinine equation: more accurate GFR estimates, lower CKD prevalence estimates, and better risk predictions. Am J Kidney Dis 2010;55:622-7.

21 Mueller C, McDonald K, de Boer RA, et al. Heart failure association of the European Society of cardiology practical guidance on the use of natriuretic peptide concentrations. Eur J Heart Fail 2019;21:715-31.

22 von Elm E, Altman DG, Egger M, et al. The strengthening the reporting of observational studies in epidemiology (STROBE) statement: guidelines for reporting observational studies. Lancet 2007;370:1453-7.

23 D'Alonzo GE, Barst RJ, Ayres SM, et al. Survival in patients with primary pulmonary hypertension. Results from a national prospective registry. Ann Intern Med 1991;115:343-9.

24 Lewis JF, Webber JD, Sutton LL, et al. Discordance in degree of right and left ventricular dilation in patients with dilated cardiomyopathy: recognition and clinical implications. J Am Coll Cardiol 1993;21:649-54.

25 Mutlak D, Aronson D, Lessick J, et al. Functional tricuspid regurgitation in patients with pulmonary hypertension: is pulmonary artery pressure the only determinant of regurgitation severity? Chest 2009; 135:115-21.

26 Mehta PA, Cowie MR. Gender and heart failure: a population perspective. Heart 2006;92(Suppl 3):iii14-18.

27 Melenovsky V, Kotrc M, Borlaug BA, et al. Relationships between right ventricular function, body composition, and prognosis in advanced heart failure. J Am Coll Cardiol 2013;62:1660-70.

28 Tepsuwan T, Schuarattanapong S, Woragidpoonpol S, et al. Incidence and impact of cardiac cachexia in valvular surgery. Asian Cardiovasc Thorac Ann 2009;17:617-21.

29 Seko Y, Kato T, Haruna T, et al. Association between atrial fibrillation, atrial enlargement, and left ventricular geometric remodeling. Sci Rep 2018;8:6366.

30 Kiehl EL, Makki T, Kumar R, et al. Incidence and predictors of right ventricular pacing-induced cardiomyopathy in patients with 
complete atrioventricular block and preserved left ventricular systolic function. Heart Rhythm 2016;13:2272-8.

31 Lankeit M, Keller K, Tschöpe C, et al. [Medicinal treatment of tricuspid valve regurgitation]. Herz 2017;42:634-43.
32 Topilsky Y, Khanna AD, Oh JK, et al. Preoperative factors associated with adverse outcome after tricuspid valve replacement. Circulation 2011;123:1929-39.

33 Arsalan M, Walther T, Smith RL, et al. Tricuspid regurgitation diagnosis and treatment. Eur Heart J 2017;38:634-8. 